\title{
Prognostic utility of pre- and postoperative plasma L-lactate measurements in hospitalized cows with acute abdominal emergencies
}

\author{
S. Giertzuch, ${ }^{1} \odot$ A. Lorch, ${ }^{1}$ C. K. Lausch, ${ }^{1} \odot$ G. Knubben-Schweizer, ${ }^{1}$ (1) and F. M. Trefz ${ }^{1,2 *}$ () \\ ${ }^{1}$ Clinic for Ruminants with Ambulatory and Herd Health Services at the Centre for Clinical Veterinary Medicine, Ludwig-Maximilians-Universität \\ (LMU) Munich, Sonnenstrasse 16, 85764 Oberschleißheim, Germany \\ ${ }^{2}$ Clinic for Ruminants, Vetsuisse-Faculty, University of Bern, Bremgartenstrasse 109a, 3012 Bern, Switzerland
}

\begin{abstract}
The aim of the present analyses was to compare the prognostic value of pre- and postoperative L-lactate measurements in hospitalized cows requiring surgical intervention for an acute abdominal emergency, such as gastrointestinal ileus or peritonitis. For this purpose, we analyzed data from retro- and prospective case series, consisting of 754 and 98 cows, respectively. Plasma Llactate concentrations (L-LAC) were determined upon admission to the hospital (both study populations), immediately before initiation of surgical intervention (prospective study population), and 6, 12, 24, 48, and $72 \mathrm{~h}$ later (prospective study population). The outcome of cows was evaluated until hospital discharge (both study populations) and 3 mo after discharge by a phone call to the farmer (prospective study population). A negative outcome was defined as death or euthanasia during hospitalization, or if discharged animals had an unsatisfied owner or were culled for medical reasons that were directly related to the initial abdominal emergency. For the retrospective study population, the overall survival rate until hospital discharge was $66 \%$. Cows with a negative outcome (median: $6.81 \mathrm{mmol} / \mathrm{L}$ ) had significantly higher L-LAC than cows with a positive outcome $(3.66 \mathrm{mmol} / \mathrm{L})$ of therapy. At the individual diagnosis level, L-LAC was associated with mortality in cows with a diagnosis of abomasal volvulus, local peritonitis, hemorrhagic bowel syndrome, and jejunal volvulus. Considering the whole study population, the area under the receiver operating characteristic curve was 0.66 . For the prospective study population, the proportion of cows with a positive outcome was $65 \%$ until hospital discharge and $61 \%$ after the 3 -mo observation period. At all sampling times, before and during the first $12 \mathrm{~h}$ after surgical intervention, cows with a negative outcome had significantly higher L-
\end{abstract}

Received June 17, 2020.

Accepted July 2, 2020.

*Corresponding author: florian.trefz@vetsuisse.unibe.ch
LAC than cows with a positive outcome. The largest area under the receiver operating characteristic curve for L-LAC was observed at $6 \mathrm{~h}(0.89)$. A cut-point of $1.77 \mathrm{mmol} / \mathrm{L}$ was identified, which had a sensitivity and specificity for predicting a negative outcome until hospital discharge of 88.9 and $73.4 \%$, respectively. The present analyses confirmed previous findings in calves and show that persistent hyper-L-lactatemia during the early postoperative period is a more reliable indicator for a negative outcome than hyper-L-lactatemia before initiation of surgical intervention.

Key words: cow, gastrointestinal ileus, peritonitis, hyper-L-lactatemia, mortality

\section{INTRODUCTION}

A variety of diseases, such as right-sided abomasal disorders, hemorrhagic bowel syndrome, intestinal intussusceptions or strangulations, and peritonitis, can result in the clinical picture of an acute abdominal emergency in cows, which is characterized by varying degrees of abdominal distension, circulatory or peristaltic disorders, and abdominal pain. To save the animal and to verify the suspected diagnosis, these conditions usually require immediate surgical intervention. However, the proportion of animals with a negative outcome after surgical intervention was reported to be $63 \%$ in cows with hemorrhagic bowel syndrome (Braun et al., 2010), 57\% in cattle with intestinal intussusceptions (Constable et al., 1997), and range from $26.3 \%$ to $65 \%$ in cows with abomasal volvulus (Constable et al., 1991; Figueiredo et al., 2006; Boulay et al., 2014). For reasons including high therapeutic expenditures and animal welfare, there is an interest in objective, detectable markers that allow for an accurate prediction of the outcome of therapy in affected animals before initiation, or at least at an early stage of therapy.

Previous studies have mainly focused on prognostic factors in cows with right-sided displacements of the abomasum and hypochloremia, hyponatremia, increased anion gap, and azotemia, as well as clinical findings 
such as tachycardia and dehydration were reported to be associated with an increased risk for a negative outcome in these animals (Constable et al., 1991; Fubini et al., 1991; Meylan, 1999). Because L-lactate (L-LAC) is a well-established biomarker of tissue hypoxia, sepsis, and disease severity, measurement of this metabolite has also attracted increasing attention in bovine medicine due to the availability and validation of cheap and portable analyzers (Burfeind and Heuwieser, 2012; Karapinar et al., 2013; Buczinski et al., 2014). Cow-side measurements of blood L-LAC allow for the prediction of a negative outcome of therapy in cows with right abomasal displacements (Boulay et al., 2014; Buczinski et al., 2015), which makes L-LAC a potentially useful prognostic indicator for other abdominal emergencies in cattle, as well. However, a recent retrospective study involving 587 hospitalized calves suffering from acute abdominal emergencies of different causes indicated that a single preoperative determination of L-LAC has limited clinical utility for predicting nonsurvival until hospital discharge because the respective prognostic information depends on the intraoperative diagnosis (Lausch et al., 2019). More importantly, a subsequent prospective investigation revealed that persistent hyper-L-lactatemia during the initial therapeutic period represents a more reliable prognostic indicator than hyper-L-lactatemia before initiation of surgical intervention (Lausch et al., 2020).

The aim of the present study was to perform similar investigations in hospitalized cows with a broad spectrum of surgical abdominal emergencies. Based on previous findings in calves, we hypothesized that preoperative L-LAC measurements provide limited prognostic information in affected cows as well, but that determination of L-LAC during the early postoperative period is a clinically useful monitoring tool that can help to identify animals with a high mortality risk. We aimed to verify this hypothesis by means of retro- and prospective investigations.

\section{MATERIALS AND METHODS}

\section{Retrospective Case Series}

To assess the prognostic utility of preoperative LLAC measurements in a large study population with a broad spectrum of acute abdominal emergencies, the medical records of 754 cows admitted to the Clinic for Ruminants with Ambulatory and Herd Health Services of the Ludwig-Maximilians-Universität (LMU) Munich between May 2005 and November 2012 were analyzed. This time period was selected because it provided the largest number of cases during which laboratory parameters were determined with the same laboratory equip- ment. Cows were selected for the analysis if plasma L-LAC, as part of a routinely performed biochemistry panel, was measured upon admission, and a subsequent surgical intervention during the first hours of hospitalization for an acute abdominal emergency (including all cases of abomasal disorders, suspected gastrointestinal ileus, or peritonitis) was planned or eventually carried out. Information retrieved from the medical records included age, sex, breed, surgical procedures, intraoperative diagnoses, outcome, and the results of biochemical analyses. Similar to our previous analysis in calves (Lausch et al., 2019), grouping of cows was carried out by the location of the underlying problem (abomasum, small intestine, large intestine, multiple parts of the gastrointestinal tract, or abdominal cavity), as well as by the major clinical diagnosis. The latter was determined based on the documented clinical and intraoperative findings and was considered to be the single most detrimental diagnosis that was responsible for the clinical picture and the condition of the cow, even if other contributing problems were present.

\section{Prospective Case Series}

To comparatively assess the prognostic utility of pre- and postoperative L-LAC measurements, 98 cows that were admitted to the Clinic for Ruminants with Ambulatory and Herd Health Services, LMU Munich between August 20, 2015 and August 20, 2016 were included in a subsequent prospective observational study. Every cow that was admitted during this time period was included in the study if she suffered from an acute abdominal emergency and required immediate surgical intervention for an abomasal disorder, suspected gastrointestinal ileus, or peritonitis. Cows with abdominal emergencies that were eligible for conservative treatment were not included in the study. This investigation was approved by the Ethics committee of the Centre of Veterinary Clinical Medicine, LMU Munich (permit No. 39-15-01-2015).

\section{Clinical Management and Treatment of Cows}

After admission to the hospital, cows underwent a thorough clinical examination, which included an ultrasonographic examination if deemed necessary, by the responsible clinician. For the prospective investigation, cows were weighed and a 16-gauge catheter (Cavafix Certo with Splittocan, B. Braun, Melsungen, Germany) was placed in a jugular vein and secured in place with suture material. For catheter placement, the area over the respective jugular vein was clipped and antiseptically prepared. Then, $2 \mathrm{~mL}$ of a $2 \%$ procaine solution was injected subcutaneously before 
catheterization. Surgical intervention was carried out by means of a right flank laparotomy under local anesthesia in a standing position or left lateral recumbency if the underlying problem could not be corrected in a standing position (or in the case of impairments of ability to stand). Perioperative treatment consisted of intravenous fluid therapy with constant drip infusions containing saline, glucose, and calcium gluconate infusion solutions, as well as administration of antibiotics and nonsteroidal drugs.

After surgical intervention, cows were treated orally with sodium sulfate $(1 \mathrm{~g} / \mathrm{kg}$ of body mass) and received potassium chloride depending on the determined plasma potassium concentration upon admission. Furthermore, cows were treated with neostigmine (Konstigmin, Vetoquinol GmbH, Ismaning, Germany) at a dosage of 0.025 $\mathrm{mg} / \mathrm{kg}$ of body mass subcutaneously if deemed necessary by the responsible clinician. Neostigmin administration was initiated when the peritoneum was closed and administered at least 4 times in 3-h intervals. For the prospective investigation, treatment was standardized as much as possible as outlined in Supplemental Table S1 (https://doi.org/10.3168/jds.2020-19102), but any additional treatment or minor deviations from the treatment protocol were at the discretion of the responsible clinician when deemed necessary in a specific case.

\section{Blood Sampling and Laboratory Analyses}

Concentrations of L-LAC were determined from heparinized blood samples containing potassium fluoride to inhibit glycolysis. During the prospective study, blood samples were collected from the catheter upon admission, immediately before initiation of surgery $(0 \mathrm{~h})$, and $6,12,24,48$, and $72 \mathrm{~h}$ later. Within $30 \mathrm{~min}$ after collection, samples were centrifuged at room temperature at $1,500 \times g$ for $5 \mathrm{~min}$. For the retrospective study population, blood samples for determination of L-LAC were collected from the jugular vein by venipuncture on admission.

Plasma samples were analyzed by means of automatic systems [Hitachi 911 (retrospective study) and Cobas c 311 (prospective study) analyzers; Roche Diagnostics, Mannheim, Germany] using the same enzymatic test reaction (Shimojo et al., 1989) with a minimum limit of quantification of $0.17 \mathrm{mmol} / \mathrm{L}$. Hyper-L-lactatemia was defined as a plasma L-LAC > $2.2 \mathrm{mmol} / \mathrm{L}$ (Rosenberger, 1990).

During the prospective investigation, intravenous fluid therapy was initiated preoperatively after admission blood samples were taken if signs of marked clinical dehydration were evident. Infusions were stopped 15 min before each blood sampling, and the administered amount of fluid per kilogram of body mass was calculated and recorded.

\section{Outcome of Therapy}

In respect to the retrospective case series, a positive outcome (PO; survival) was defined as discharge from the hospital. The group of animals with a negative outcome (NO; nonsurvival) consisted of cows that died or were euthanized during hospitalization because of fatal intraoperative findings, massive deterioration in general condition, ongoing signs of gastrointestinal ileus, or animal welfare reasons.

For the purpose of the prospective study, every clinician was blinded to the postoperative course of L-LAC, and a decision to euthanize a cow was exclusively made on the basis of clinical or intraoperative findings. Economic reasons were not considered in the decision to euthanize a cow and, if the general condition of the cow had allowed, a re-laparotomy was performed before euthanasia. Cows were discharged from the hospital when the feed intake and general condition had normalized. For this subset of animals, 2 outcome definitions were used. The outcome of cows was evaluated until hospital discharge and additionally after 3 mo following discharge by means of a phone call to the farmer. During the telephone call, the farmers were asked to provide the following information: whether the cow was still alive, whether she was still in the herd, and whether the owner was satisfied with the animal's postsurgical progress. If the cow was culled, the owner was asked when she was culled, the reason for culling, and whether culling was related to the initial acute abdominal problem (Boulay et al., 2014). The interviews were performed by the same person (S. G.), and every farmer was asked the same questions in the same order using a standardized protocol. Subsequently, cows were allocated to the group of cows with a PO if discharged cows were still in the herd, had a satisfied owner, or were culled despite a positive postsurgical evolution for reasons that were not directly related to the initial abdominal emergency problem. In contrast, a NO of therapy was determined by death or euthanasia during hospitalization, if discharged animals had an unsatisfied owner, or if animals were culled for medical reasons that were directly related to the initial abdominal emergency problem.

\section{Statistical Analysis}

Statistical analysis was performed using Microsoft Excel (version 2010, Microsoft Corporation, Redmond, WA), SPSS (version 24.0, IBM, New York, NY), and GraphPad Prism (version 7.01, GraphPad Software, 
La Jolla, CA). The $P$-values $<0.05$ were considered statistically significant. A normal distribution of data was assessed by the Shapiro-Wilk test and visual inspection of quantile-quantile plots. Because most of the data were not normally distributed, nonparametric tests were used, and data are presented as medians and interquartile ranges $\left(Q_{1}-Q_{3}\right)$. Spearman's coefficients of correlation were calculated to determine associations between parameters.

For the prospective data set, 2 different methods were used to assess the dynamics of L-LAC over time. The percentage change of plasma L-LAC concentrations $\left(\Delta_{\%} \mathrm{~L}-\mathrm{LAC}\right)$ in relation to values upon admission $\left(\mathrm{L}-\mathrm{LAC}_{\mathrm{Adm}}\right)$ was calculated as follows: $\Delta_{\%} \mathrm{~L}-\mathrm{LAC}=$ $\left(\mathrm{L}-\mathrm{LAC}_{\text {hour x }}-\mathrm{L}_{-} \mathrm{LAC}_{\mathrm{Adm}}\right) / \mathrm{L}-\mathrm{LAC}_{\mathrm{Adm}} \times 100$. Furthermore, the area under the L-LAC concentration-time curve $\left(\mathrm{L}-\mathrm{LAC}_{\mathrm{AUC}}\right)$ was calculated for different time intervals using the trapezoid method (Wilkins et al., 2015). For this purpose, the area for each trapezoid between 2 consecutive sampling times was calculated and summed if necessary to obtain the total L- $\mathrm{LAC}_{\mathrm{AUC}}$ for the respective time interval of interest.

Mann-Whitney $U$-tests were used to determine statistically significant differences of L-LAC, $\Delta_{\%} \mathrm{~L}-\mathrm{LAC}$, and $\mathrm{L}-\mathrm{LAC}_{\mathrm{AUC}}$ between cows with PO and NO. Furthermore, the predictive ability of these variables was assessed by means of a receiver operating characteristic (ROC) analysis. This included the calculation of the area under the ROC curve (AUC-ROC) and the associated $95 \%$ confidence interval, as well as the identification of cut-off values that optimized the resulting sensitivity and specificity for the prediction of a NO on the basis of the Youden index (Youden, 1950). For the prospective data set, the association between LLAC and a NO after 3 mo following discharge was also evaluated using binary logistic regression analysis. For this purpose, odds ratios for every $\mathrm{mmol} / \mathrm{L}$ increase of L-LAC with associated $95 \%$ confidence intervals were calculated for each sampling time. For the retrospective data set, survival rates in relation to deciles of plasma L-LAC were additionally evaluated. For this purpose, a chi-squared test was used to compare survival rates of each decile to the survival rate of cows of the decile that best lay within the reference range (Trefz et al., 2017). In this case, the level of significance was adjusted using the Bonferroni method $(P<0.006)$.

\section{RESULTS}

\section{Retrospective Case Series}

Study Population. Most cows were German Fleckvieh $(79.4 \%, \mathrm{n}=599)$, which is the most common dairy breed in Bavaria. The remaining breeds were HolsteinFriesian $(10.1 \%, \mathrm{n}=76)$, German Braunvieh (6.5\%, $\mathrm{n}=49)$, crossbreds $(3.2 \%, \mathrm{n}=24)$, Pinzgau $(\mathrm{n}=1)$, and undocumented in 5 cows. The median age (and interquartile range) was $4.4(3.4-5.7)$ yr. All but 69 cows were in lactation.

Diagnoses and Outcome of Therapy. The overall survival rate until hospital discharge was $66 \%$ ( $\mathrm{n}=$ 498). A total of 256 cows did not survive until hospital discharge, of which 27 cows died spontaneously and 229 cows were euthanized. Eight cows had to be euthanized due to a massive deterioration in general condition or agonal state before surgical intervention. In these cows, the underlying disorder was diagnosed by a necropsy examination. A total of 106 cows had a NO during surgery, whereas 142 cows died or were euthanized after surgery. The median length of hospitalization (and interquartile range) for cows with a PO was $5(4-7)$ days. Individual diagnoses and respective survival rates of cows are listed in Table 1. A total of 385 cows (51\%) suffered from an abomasal disorder, 186 cows (24.7\%) had a problem located in the small intestine, and 79 cows $(10.5 \%)$ had a problem in the large intestine. Multiple parts of the gastrointestinal tract were affected in 23 cows $(3.1 \%)$, and 80 cows $(10.6 \%)$ suffered from intraabdominal adhesions or peritonitis. Generalized peritonitis was diagnosed in 49 cows and was considered related to perforated abomasal ulcers $(n=23)$, necrosis or perforation of organs $(n=10)$, reticular foreign bodies $(\mathrm{n}=6)$, perforation of the uterus $(\mathrm{n}=$ $3)$, and undetermined $(\mathrm{n}=7)$.

Association Between L-LAC and Outcome of Therapy. Hyper-L-lactatemia, defined as plasma LLAC $>2.2 \mathrm{mmol} / \mathrm{L}$, was present in 577 cows $(76.5 \%)$. Plasma L-LAC were higher $(P<0.001)$ in cows with a NO (median: 6.81; $\mathrm{Q}_{1}-\mathrm{Q}_{3}: 3.44-9.98 \mathrm{mmol} / \mathrm{L}$ ) than in cows with a PO of therapy (median: $3.66 ; \mathrm{Q}_{1}-\mathrm{Q}_{3}$ : $2.04-6.99 \mathrm{mmol} / \mathrm{L}$ ). The proportion of cows with a NO in deciles of plasma L-LAC is shown in Figure 1. Only the survival rates of cows in the ninth and tenth decile were significantly different from those of cows in the reference decile. The AUC-ROC curve was $0.66(95 \%$ CI: $0.62-0.70, P<0.001)$. An identified cut-point for plasma L-LAC of $5.37 \mathrm{mmol} / \mathrm{L}$ had a sensitivity and specificity for the prediction of nonsurvival of $61.3 \%$ and $65.3 \%$, respectively.

Prognostic Value of Plasma L-LAC Stratified by Intraoperative Diagnoses. Plasma L-LAC stratified by major clinical diagnoses and respective values for plasma L-LAC for cows with a $\mathrm{PO}$ or NO of therapy are also shown in Table 1 . The highest values for plasma L-LAC were seen in cows with impaction with coagulated blood (hemorrhagic bowel syndrome) 
Giertzuch et al.: L-LACTATE IN COWS WITH ACUTE ABDOMINAL EMERGENCIES

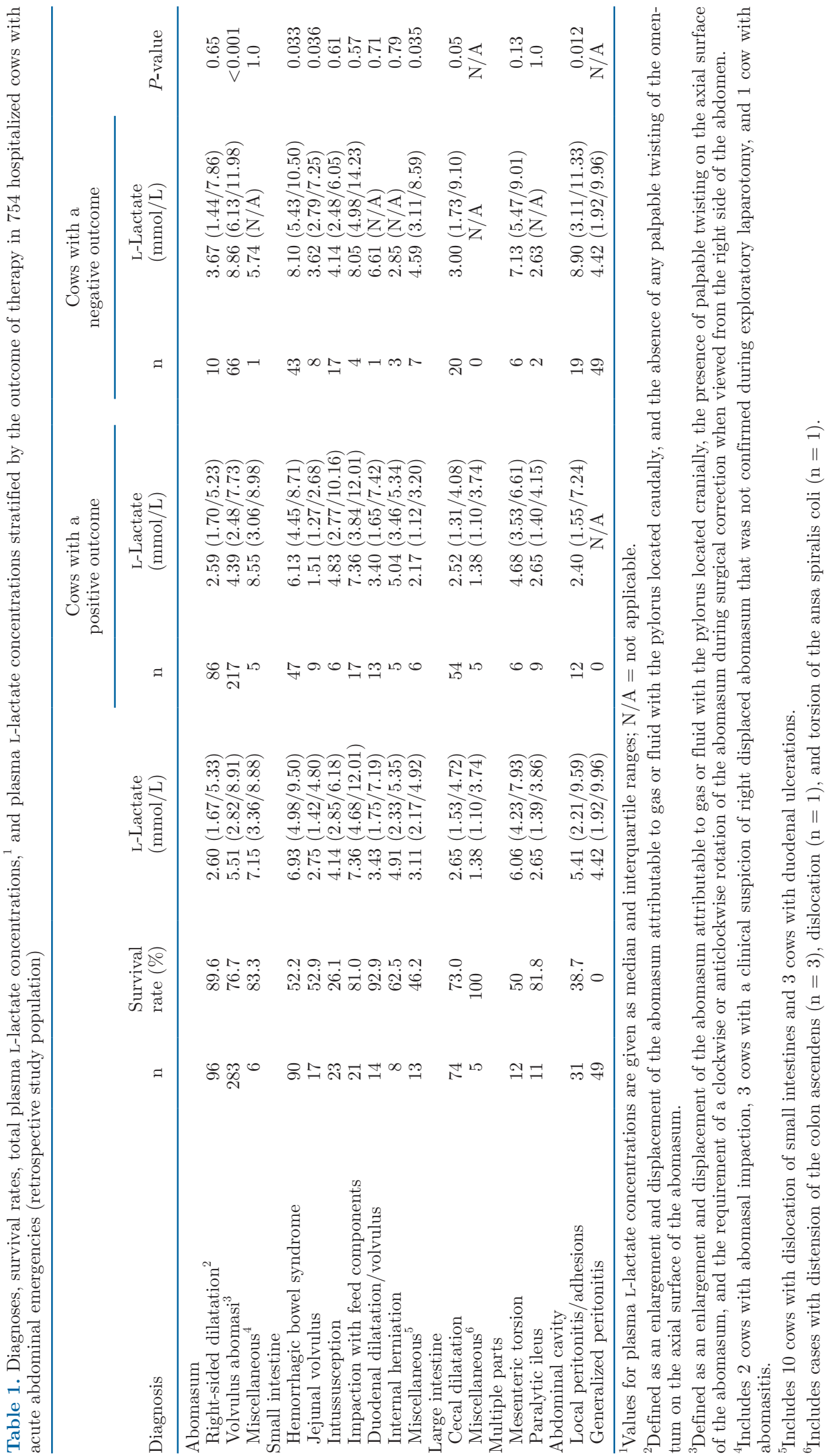


or feed components, as well as in cases with mesenteric torsion. A statistically significant difference in plasma L-LAC between cows with a PO and NO was found for cows with abomasal volvulus, volvulus jejuni, hemorrhagic bowel syndrome, and local peritonitis or adhesions. Results of a ROC analysis that assessed the diagnostic accuracy of preoperative plasma L-LAC for the prediction of nonsurvival in those groups of cows, including a group with all kinds of right-sided abomasal disorders, are presented in Table 2.

\section{Prospective Case Series}

Study Population. Most of the cows ( $\mathrm{n}=87 ; 88.8 \%)$ belonged to the German Fleckvieh breed. Seven cows were Holstein-Friesian, 2 cows were German Braunvieh, and 2 cows were crossbreds. The median value $\left(\mathrm{Q}_{1}-\mathrm{Q}_{3}\right)$ for age was $4.1(3.1-5.0)$ yr. All but 8 cows were in lactation.

Diagnoses and Outcome of Therapy. Individual diagnoses and the respective number of affected cows are reported in Table 3. Right-sided abomasal disorders and hemorrhagic bowel syndrome were the most frequent diagnoses. As shown in Figure 2, a total of 64 cows (65.3\%) survived until hospital discharge. A total of 34 cows $(34.7 \%)$ had a NO during hospitalization, of which 29 cows were euthanized and 5 cows died spontaneously. Twelve cows were euthanized during the initial surgical intervention due to generalized peritonitis, perforated abomasal ulcers, and rupture or necrosis of the intestinal wall. Another 17 cows were euthanized during the subsequent course of hospitalization due to ongoing or recurrent signs of gastrointestinal ileus or a massive deterioration of the general condition. A relaparotomy was performed in a total of 11 cows.

Follow-up interviews revealed that 8 out of 64 discharged cows had been removed from their herds during the 3-mo period. Four cows were reported to be culled due to a relapse with signs of colic, reduced feed intake, and milk yield between 4 and 11 wk following discharge. The initial diagnoses in those 4 cows

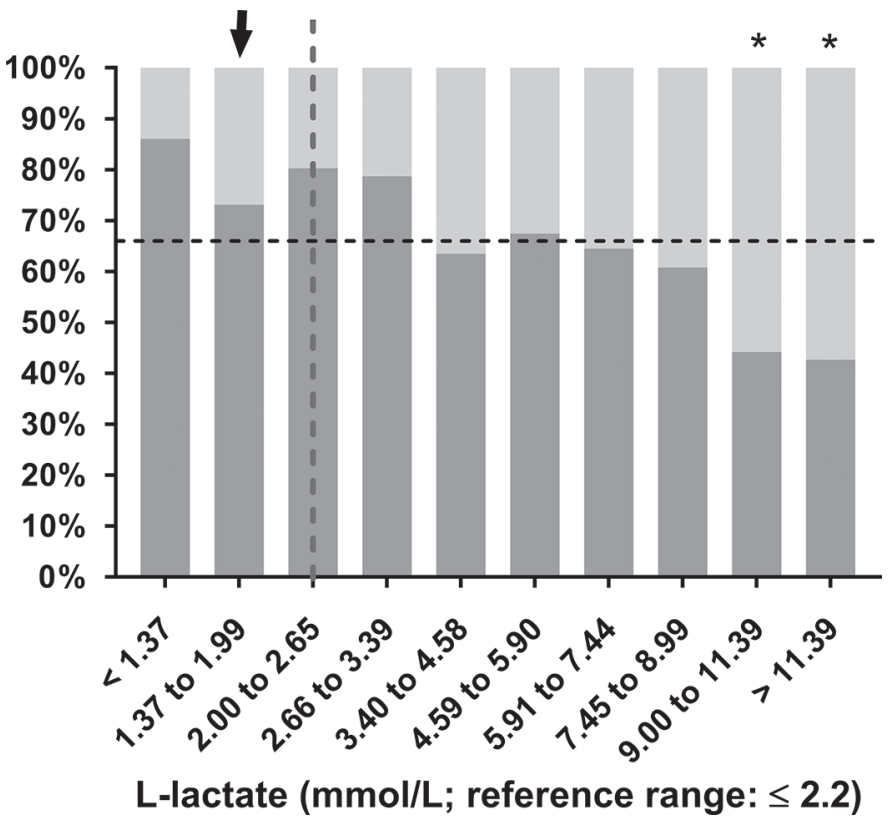

Figure 1. Survival rates (dark gray bars) of 754 cows with acute abdominal emergencies in deciles of plasma L-lactate concentrations (retrospective study population). The dashed vertical line indicates the reference range for plasma L-lactate concentration and the dashed horizontal line indicates the overall survival rate of cows of this study population $(66 \%)$. Survival rates of decile groups that were significantly different $(P<0.006)$ from the survival rate of the reference group (arrow) are indicated by asterisks.

were hemorrhagic bowel syndrome (3 cows of which 1 cow had a concomitant intussusception) and duodenal sigmoid flexure volvulus (1 cow). These 4 cows were therefore allocated to the group of cows with a NO. Three additional cows were culled due to economic reasons despite a satisfactory postsurgical evolution, and 1 cow was euthanized for reasons of a limb fracture. These 4 cows remained in the group of cows with a PO. Therefore, the total number of cows with a PO after the 3 -mo observation period was $60(61.2 \%)$.

Association Between Plasma L-LAC Concentrations and Outcome of Therapy. Plasma L-LAC in cows with $\mathrm{PO}$ and $\mathrm{NO}$ at respective sampling times

Table 2. Results of a receiver operating characteristic analysis assessing the diagnostic accuracy of plasma L-lactate concentrations for the prediction of nonsurvival in cows with a diagnosis of abomasal volvulus, all types of right-sided abomasal disorders, hemorrhagic bowel syndrome, jejunal volvulus, and local peritonitis or adhesions (retrospective study population)

\begin{tabular}{|c|c|c|c|c|c|c|c|}
\hline Diagnosis & $\mathrm{n}$ & $\mathrm{AUC}^{1}$ & $\begin{array}{l}95 \% \mathrm{CI} \\
\text { for AUC }\end{array}$ & $P$-value & $\begin{array}{c}\text { Cut-off for } \\
\text { L-lactate }(\mathrm{mmol} / \mathrm{L})\end{array}$ & $\begin{array}{l}\text { Sensitivity } \\
(\%)\end{array}$ & $\begin{array}{l}\text { Specificity } \\
(\%)\end{array}$ \\
\hline Volvulus abomasi & 283 & 0.76 & $0.69-0.82$ & $<0.001$ & 5.24 & 84.8 & 58.1 \\
\hline Right abomasal disorders & 379 & 0.74 & $0.68-0.80$ & $<0.001$ & 5.24 & 77.6 & 63.0 \\
\hline Hemorrhagic bowel syndrome & 90 & 0.63 & $0.52-0.75$ & 0.033 & 6.53 & 40.4 & 59.6 \\
\hline Jejunal volvulus & 17 & 0.81 & $0.0-1.0$ & 0.034 & 2.65 & 87.5 & 77.8 \\
\hline Local peritonitis or adhesions & 31 & 0.77 & $0.60-0.94$ & 0.013 & 8.74 & 52.6 & 91.7 \\
\hline
\end{tabular}

${ }^{1} \mathrm{AUC}=$ area under the receiver operating characteristic curve. 


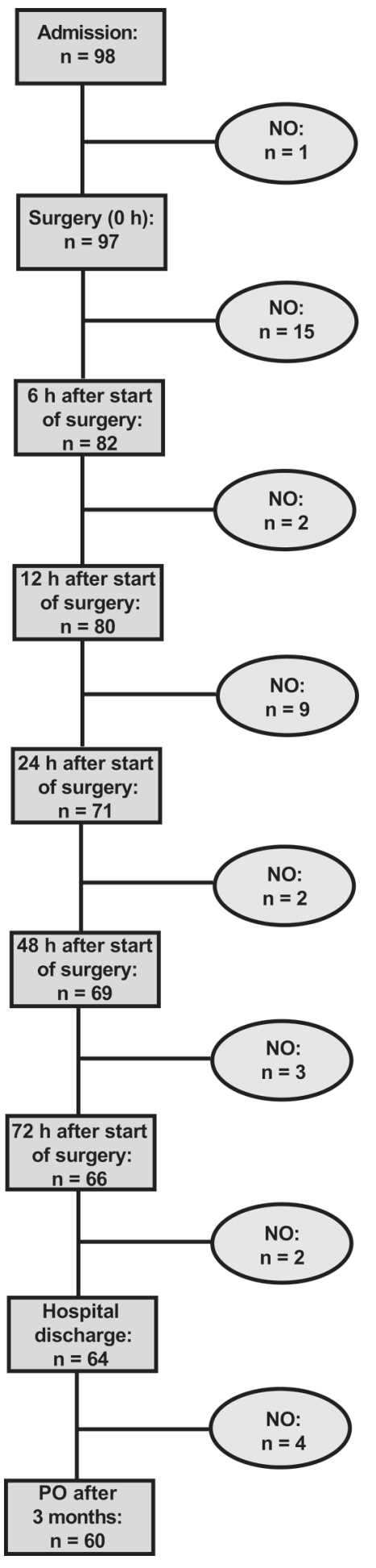

Figure 2. Flowchart illustrating the outcome of therapy of 98 hospitalized cows with acute surgical abdominal emergencies (prospective study population). Ovals to the right indicate the number of cows with a negative outcome (NO) between respective time points. The final outcome of cows was evaluated 3 mo after discharge by a phone call to the farmer, and NO was defined as death or euthanasia during hospitalization, if discharged animals had an unsatisfied owner, or were culled for medical reasons that were directly related to the initial abdominal emergency problem. $\mathrm{PO}=$ positive outcome. are reported in Table 4 . The median time interval between blood sampling upon admission and initiation of surgical intervention was $47 \mathrm{~min}$. Related to the clinical management of cows, L-LAC was not reassessed in a total of 3 cows at $0 \mathrm{~h}$ and 1 cow at $72 \mathrm{~h}$.

In terms of the outcome until hospital discharge, LLAC was significantly higher in cows with a NO than in cows with a PO at all sampling times before and the first $24 \mathrm{~h}$ after surgical intervention. In terms of the outcome during the 3-mo observation period, L-LAC was significantly higher in cows with a NO than in cows with PO at all sampling times until $12 \mathrm{~h}$ following surgery. Results of the ROC analyses and respective cutoff values that optimized the resulting sensitivity and specificity for the prediction of a NO at each sampling time are reported in Table 5. For both the outcome until hospital discharge and the outcome during the 3-mo observation period, the highest AUC-ROC values were found at $6 \mathrm{~h}$ (0.89 and 0.80$)$, which were higher than for preoperative L-LAC measurements on admission (0.69 and 0.70 ) and $0 \mathrm{~h}$ (0.71 for both outcome definitions). A binary logistic regression analysis indicated that the odds ratio for NO after 3 mo following discharge increased by a factor of 1.19 for every $\mathrm{mmol} / \mathrm{L}$ increase of

Table 3. Individual intraoperative diagnoses of cows of the prospective study population $(\mathrm{n}=98)$

\begin{tabular}{lc}
\hline Lesion location and individual diagnoses & $\begin{array}{c}\text { Number } \\
\text { of cows }\end{array}$ \\
\hline Abomasum & \\
Right-sided dilatation $^{1}$ & 10 \\
Abomasal volvulus $^{2}$ & 23 \\
Pyloric impaction $_{\text {Posterior functional stenosis }}$ & 1 \\
Small intestine $_{\text {Hemorrhagic bowel syndrome }}^{3}$ & 31 \\
Intussusception $_{\text {Duodenal dilatation or volvulus }}$ & 2 \\
Impaction with feed components $_{\text {Jejunal volvulus }}$ & 3 \\
Large intestine & 2 \\
Cecal dilatation and dislocation & 2 \\
Multiple parts & \\
Mesenteric torsion & 8 \\
Paralytic ileus & \\
Abdominal cavity & 4 \\
Peritoneal adhesions or local adhesions & 3 \\
Generalized peritonitis & 5 \\
\hline
\end{tabular}

${ }^{1}$ Defined as an enlargement and displacement of the abomasum attributable to gas or fluid with the pylorus located caudally, and the absence of any palpable twisting of the omentum on the axial surface of the abomasum.

${ }^{2}$ Defined as an enlargement and displacement of the abomasum attributable to gas or fluid with the pylorus located cranially, the presence of palpable twisting on the axial surface of the abomasum, and the requirement of a clockwise or anticlockwise rotation of the abomasum during surgical correction when viewed from the right side of the abdomen.

${ }^{3}$ Accompanied by a small intestinal intussusception in 4 cows. 
Table 4. Median and interquartile range $\left(\mathrm{Q}_{1}-\mathrm{Q}_{3}\right)$ for plasma L-lactate concentrations $(\mathrm{mmol} / \mathrm{L})$ of cows of the prospective study population stratified by sampling times and the outcome of therapy ${ }^{1}$

\begin{tabular}{|c|c|c|c|c|c|c|c|}
\hline \multirow[b]{2}{*}{ Sampling time } & \multicolumn{3}{|c|}{ Cows with a positive outcome } & \multicolumn{3}{|c|}{ Cows with a negative outcome } & \multirow[b]{2}{*}{$P$-value } \\
\hline & $\mathrm{n}$ & Median & $\mathrm{Q}_{1}-\mathrm{Q}_{3}$ & $\mathrm{n}$ & Median & $\mathrm{Q}_{1}-\mathrm{Q}_{3}$ & \\
\hline Admission & 64 & 3.23 & $1.56-7.10$ & 34 & 6.12 & $3.26-10.0$ & 0.002 \\
\hline $0 \mathrm{~h}$ & 62 & 2.32 & $1.46-5.81$ & 32 & 5.37 & $3.63-8.71$ & 0.001 \\
\hline $6 \mathrm{~h}$ & 64 & 1.36 & $0.94-2.01$ & 18 & 4.12 & $2.26-9.26$ & $<0.001$ \\
\hline $12 \mathrm{~h}$ & 64 & 1.07 & $0.88-1.29$ & 16 & 2.50 & $2.50-7.03$ & $<0.001$ \\
\hline $72 \mathrm{~h}$ & 63 & 0.65 & $0.58-0.72$ & 2 & 0.70 & $\mathrm{~N} / \mathrm{A}^{2}$ & 0.73 \\
\hline \multicolumn{8}{|c|}{ Outcome 3 mo after discharge } \\
\hline Admission & 60 & 3.08 & $1.56-6.00$ & 38 & 6.48 & $3.26-9.67$ & 0.001 \\
\hline $0 \mathrm{~h}$ & 58 & 2.28 & $1.46-5.63$ & 36 & 5.36 & $3.63-8.03$ & 0.001 \\
\hline $6 \mathrm{~h}$ & 60 & 1.36 & $0.94-2.16$ & 22 & 3.29 & $1.54-9.13$ & $<0.001$ \\
\hline $12 \mathrm{~h}$ & 60 & 1.07 & $0.86-1.29$ & 20 & 1.71 & $1.71-5.57$ & $<0.001$ \\
\hline $24 \mathrm{~h}$ & 60 & 0.76 & $0.76-0.87$ & 11 & 0.76 & $0.68-1.32$ & 0.13 \\
\hline
\end{tabular}

${ }^{1}$ Outcome was evaluated until hospital discharge and after 3 mo following discharge by means of a phone call to the farmer. A negative outcome of therapy was defined as death or euthanasia during hospitalization and if discharged animals had an unsatisfied owner, or were culled for medical reasons that were directly related to the initial abdominal emergency problem.

${ }^{2} \mathrm{~N} / \mathrm{A}=$ not applicable.

L-LAC on admission, but by a factor of 2.02 and 3.62 at $6 \mathrm{~h}$ and $12 \mathrm{~h}$ after surgical intervention, respectively (Table 6). The relationship between the predicted probability for a NO after 3 mo following discharge and L-LAC measured on admission, as well as 6 and $12 \mathrm{~h}$ relative to the start of surgical intervention, is comparatively visualized in Figure 3.

Association Between Changes of Plasma L-LAC Over Time and Outcome of Therapy. Percentage changes of L-LAC in relation to values on admission to the hospital are reported in Supplemental Table S2 (https://doi.org/10.3168/jds.2020-19102).
Cows with PO had a significantly more pronounced percentage decrease of L-LAC at 6 (both outcome definitions) and $12 \mathrm{~h}$ (outcome until hospital discharge) than cows with NO. The largest difference between cows with PO (median: $-51 \%$ ) and NO (median: $+28 \%)$ until hospital discharge was seen at $6 \mathrm{~h}(P=0.001)$.

Furthermore, for both outcome definitions, cows with NO had significantly higher values for L-LAC $\mathrm{AUC}_{\mathrm{C}}$ than cows with $\mathrm{PO}$ for respective time intervals between admission and $6 \mathrm{~h}, 6$ and $12 \mathrm{~h}$, admission and $12 \mathrm{~h}$, and admission and $24 \mathrm{~h}$ (Supplemental Table S2). However, results of the ROC analyses indicated that calculated

Table 5. Results of a receiver operating characteristic analysis for assessing the predictive accuracy of plasma L-lactate concentrations at different sampling times in terms of a negative outcome of therapy in cows of the prospective study population ${ }^{1}$

\begin{tabular}{|c|c|c|c|c|c|c|}
\hline Sampling time & $\mathrm{AUC}^{2}$ & $\begin{array}{l}95 \% \text { CI } \\
\text { for AUC }\end{array}$ & $P$-value & Cut-off & $\begin{array}{c}\text { Sensitivity } \\
(\%)\end{array}$ & $\begin{array}{c}\text { Specificity } \\
(\%)\end{array}$ \\
\hline \multicolumn{7}{|c|}{ Outcome until hospital discharge } \\
\hline Admission & 0.69 & $0.58-0.80$ & 0.002 & 3.89 & 73.5 & 62.5 \\
\hline $0 \mathrm{~h}$ & 0.71 & $0.60-0.82$ & 0.001 & 3.23 & 81.3 & 59.7 \\
\hline $6 \mathrm{~h}$ & 0.89 & $0.80-0.97$ & $<0.001$ & 1.77 & 88.9 & 73.4 \\
\hline $12 \mathrm{~h}$ & 0.85 & $0.73-0.97$ & $<0.001$ & 1.53 & 75.0 & 85.9 \\
\hline $24 \mathrm{~h}$ & 0.73 & $0.52-0.94$ & 0.047 & 0.94 & 57.1 & 84.4 \\
\hline \multicolumn{7}{|c|}{ Outcome 3 mo after discharge } \\
\hline Admission & 0.70 & $0.59-0.80$ & 0.001 & 3.89 & 73.7 & 65.0 \\
\hline $0 \mathrm{~h}$ & 0.71 & $0.60-0.82$ & 0.001 & 3.23 & 80.6 & 62.1 \\
\hline $6 \mathrm{~h}$ & 0.80 & $0.68-0.91$ & $<0.001$ & 2.22 & 68.2 & 76.7 \\
\hline $12 \mathrm{~h}$ & 0.79 & $0.66-0.91$ & $<0.001$ & 1.30 & 70.0 & 76.7 \\
\hline
\end{tabular}

${ }^{1}$ Outcome was evaluated until hospital discharge and after 3 mo following discharge by means of a phone call to the farmer. A negative outcome of therapy was defined as death or euthanasia during hospitalization and if discharged animals had an unsatisfied owner, or were culled for medical reasons that were directly related to the initial abdominal emergency problem.

${ }^{2} \mathrm{AUC}=$ area under the receiver operating characteristic curve. 
values for $\Delta_{\%} \mathrm{~L}-\mathrm{LAC}$ and $\mathrm{L}-\mathrm{LAC} \mathrm{AUC}_{\mathrm{AU}}$ did not provide an advantage in terms of the predictability of a $\mathrm{NO}$ over absolute values for L-LAC at the start or at the end of the respective time interval (Supplemental Table S3, https://doi.org/10.3168/jds.2020-19102).

Association of Infusion Volumes With Plasma $\boldsymbol{L}-\boldsymbol{L} \boldsymbol{A} \boldsymbol{C}$. A total number of 62 cows received intravenous fluid therapy before initiation of surgical intervention. The median infused volume until $0 \mathrm{~h}$ was $0.3 \mathrm{~L}$. Coefficients of correlation between L-LAC and $\Delta_{\%} \mathrm{~L}-\mathrm{LAC}$ at respective sampling times and the amount of fluids per kilogram of body mass that were administered during respective time intervals are reported in Supplemental Table S4 (https://doi.org/10.3168/jds.2020-19102). No significant associations were seen at 6 and $12 \mathrm{~h}$, but the administered amounts of fluids were weakly correlated with L-LAC at $24 \mathrm{~h}$ and $\Delta \% \mathrm{~L}-\mathrm{LAC}$ at 48 and $72 \mathrm{~h}$, respectively.

\section{DISCUSSION}

The present study confirms findings of our previous analyses in calves, which indicated that the diagnostic accuracy of a single preoperative L-LAC measurement depends on the intraoperative diagnosis; therefore it has limited clinical utility for the preoperative prediction of a NO (Lausch et al., 2019; Lausch et al., 2020). More importantly, our analyses confirmed our initial hypothesis that hyper-L-lactatemia during the initial postoperative period is associated with an increased risk for a NO and provides more accurate prognostic information than a single measurement before initiation of therapy.

Similar to calves with acute abdominal emergencies, a possible explanation for the limited prognostic utility

Table 6. Results of a univariable binary logistic regression analysis for assessing the association between plasma L-lactate concentrations $(\mathrm{mmol} / \mathrm{L})$ of cows of the present study population at different sampling times and a negative outcome ${ }^{1}$ of therapy after a 3-mo observation period

\begin{tabular}{lcccc}
\hline $\begin{array}{l}\text { Sampling } \\
\text { time }\end{array}$ & $\mathrm{n}$ & $\mathrm{OR}^{2}$ & $\begin{array}{l}95 \% \mathrm{CI} \\
\text { for OR }\end{array}$ & $P$-value \\
\hline Admission & 98 & 1.19 & $1.06-1.32$ & 0.003 \\
$0 \mathrm{~h}$ & 94 & 1.26 & $1.09-1.45$ & 0.002 \\
$6 \mathrm{~h}$ & 82 & 2.02 & $1.30-3.12$ & 0.002 \\
$12 \mathrm{~h}$ & 80 & 3.62 & $1.45-9.03$ & 0.006 \\
$24 \mathrm{~h}$ & 71 & 5.71 & $0.93-35.13$ & 0.06 \\
$48 \mathrm{~h}$ & 69 & 18.54 & $0.76-454.0$ & 0.074 \\
$72 \mathrm{~h}$ & 65 & 4.43 & $0.27-72.9$ & 0.30 \\
\hline
\end{tabular}

${ }^{1} \mathrm{~A}$ negative outcome of therapy was defined as death or euthanasia during hospitalization and if discharged animals had an unsatisfied owner, or were culled for medical reasons that were directly related to the initial abdominal emergency problem.

${ }^{2} \mathrm{OR}=$ odds ratio.
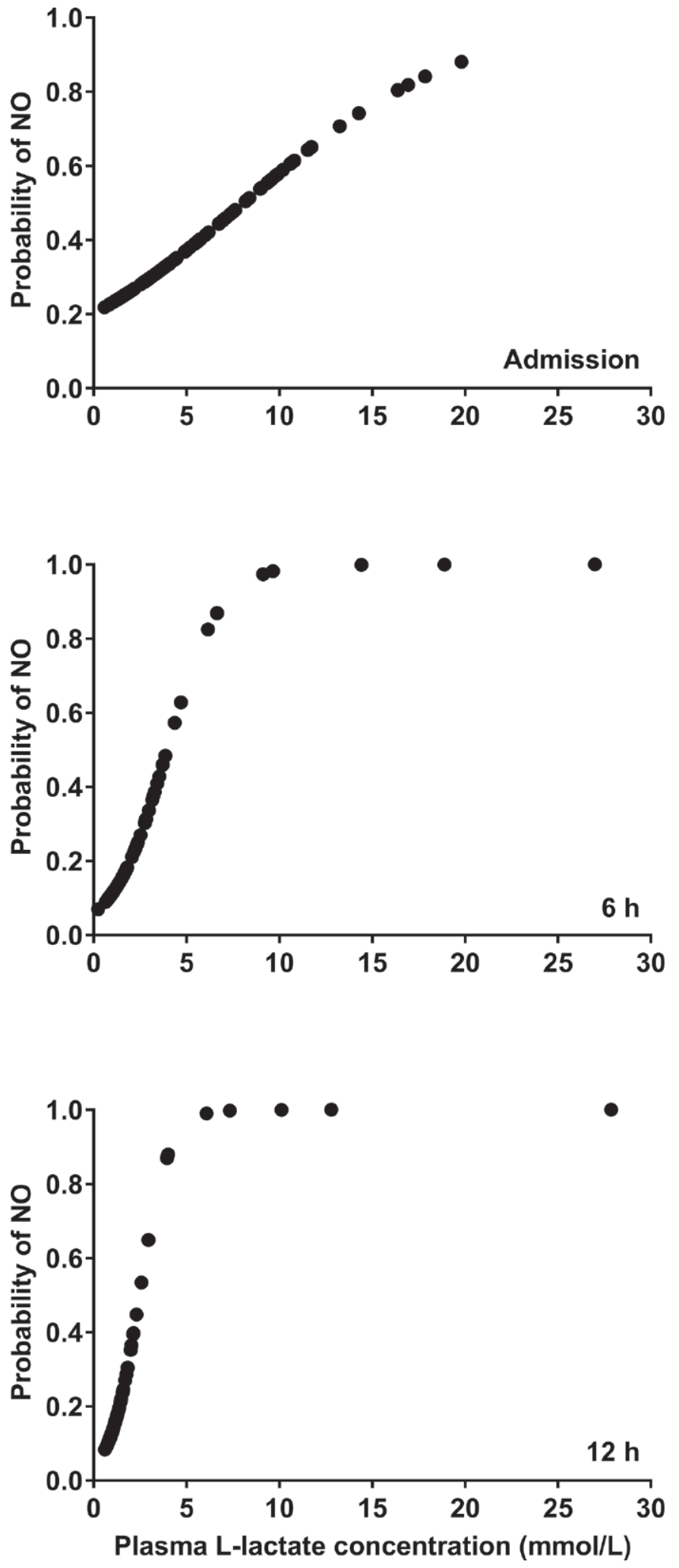

Figure 3. Relationship between plasma L-lactate concentrations of cows of the prospective study population measured on admission to the hospital, $6 \mathrm{~h}$, and $12 \mathrm{~h}$ relative to the onset of surgical intervention and the predicted probability of a negative outcome (NO) of therapy at the end of a 3-mo observation period. 
of a single preoperative L-LAC measurement is the variety of potential pathophysiological events that likely contribute to hyper-L-lactatemia in a preoperative situation, but that might have different consequences in terms of the outcome of therapy. Systemic or peripheral hypoperfusion due to hypovolemia, the presence and extent of hypovolemic shock, and the presence and extent of potential gastrointestinal ischemia are all considered mechanisms for hyper-L-lactatemia due to insufficient tissue oxygen supply (classified as type A hyperlactatemia), and might be relevant in this context (Pang and Boysen, 2007; Rosenstein et al., 2018). Adrenergic stimulation by catecholamines can be an additional cause of clinical hyper-L-lactatemia (Rosenstein et al., 2018). Therefore, stress due to transportation or in-hospital diagnostic procedures might have been another contributor to increased L-LAC after admission to the hospital.

Notably, markedly increased values for L-LAC were seen in cows with hemorrhagic bowel syndrome; values were even higher than in cows suffering from any strangulating disorders such as mesenteric torsion, volvulus jejuni, or abomasal volvulus (Table 1). This condition has become the most common cause for small intestinal ileus in recent years at our hospital. It is characterized by obstruction of the intestinal lumen with blood clots following an intensive intestinal hemorrhage due to bleeding intestinal ulcers (Rademacher et al., 2002). Multiple terms for this condition exist in the literature, including jejunal hemorrhage syndrome, or jejunal hematoma (Braun et al., 2010; Elhanafy et al., 2013; Adaska et al., 2014). The etiology of this disorder is still not completely understood. It has been discussed that Clostridium perfringens type A and Aspergillus fumigatus may be involved in the pathogenesis (Elhanafy et al., 2013), although a clear proof of evidence for involvement of these pathogens is still lacking. The disease has an acute to peracute nature, and the clinical picture of affected animals is characterized by marked depression and disturbance of the general condition (aside from signs of colic and varying degrees of dehydration and abdominal distension; Braun et al., 2010). Those findings can partly be explained by toxemia that likely results from disrupted integrity of the small intestine and a concomitant loss of the selective mucosal permeability, allowing diffusion of lipopolysaccharides and cytokines into the bloodstream (Elhanafy et al., 2013). In this context, endotoxemia might also provide an explanation for marked hyper-L-lactatemia in the absence of gastrointestinal ischemia in these animals, as endotoxemia can lead to microcirculatory dysfunction (Ince, 2005), sequestration of leukocytes with increased glucose turnover during respiratory burst (Haji-Michael et al., 1999), and altered cellular respiration known as cytopathic hypoxia (Fink, 2002; Rosenstein et al., 2018). Functional or mechanical obstruction of the intestinal tract can also potentially result in autointoxication due to prolonged intestinal transition time (Elhanafy et al., 2013; Constable et al., 2017). This might also explain a similar extent of hyper-L-lactatemia in the group of cows with small intestinal impaction due to feed components, although hypovolemia might have been another contributor in these animals.

Right-sided displacements of the abomasum and abomasal volvulus accounted for the vast majority of disorders in cows of the retrospective study population. In agreement to findings of a previous study (Boulay et al., 2014), plasma L-LAC differed significantly between surviving and nonsurviving cows with abomasal volvulus but not in cows with right-sided dilatation of the abomasum. However, the predictive accuracy of preoperative plasma L-LAC in cows with abomasal volvulus (AUC-ROC $=0.76)$ and a group of animals with all kinds of right-abomasal displacements (AUCROC $=0.74)$ was markedly lower than in the study by Boulay et al. (2014), where an AUC-ROC of 0.92 for the prediction of NO in a study population of cows with right-displaced abomasum and abomasal volvulus was reported. Those differences could be explained by differences in study design and population. The study by Boulay et al. (2014) was carried out in a bovine field service, whereas the present study was based on a university teaching-hospital population, which usually preselects more severely affected cases. This issue is reflected by L-LAC values in cows with abomasal volvulus in the present study, which were markedly higher than median L-LAC of 1.2 and $3 \mathrm{mmol} / \mathrm{L}$ in cows with PO and NO in the study by Boulay et al. (2014). Furthermore, hospitalization of cows from the present study population probably allowed for more intensive treatment and monitoring than in a field practice situation. This could have resulted in the recovery of cows with more advanced stages of the disease and, consequently, a more pronounced extent of hyper-Llactatemia. However, a major difference between these studies was the used outcome definition. In the study by Boulay et al. (2014), the outcome definition was based on the satisfaction of the owner $30 \mathrm{~d}$ postoperatively. This information was unfortunately not available for the present retrospective data set, and the outcome was consequently based on the monitoring until hospital discharge, which might be considered as a limitation.

Our prospective investigation revealed that plasma L-LAC measurements at $6 \mathrm{~h}$ after start of surgical intervention provided the most accurate prognostic information. Calculated values for $\Delta_{\%} \mathrm{~L}$-LAC further 
indicated that many cows with NO had experienced an increase of plasma L-LAC until $6 \mathrm{~h}$ after initiation of surgery, whereas a marked decrease of L-LAC was observed in cows with $\mathrm{PO}$ during the same period. Furthermore, cows with NO had significantly higher values for $\mathrm{L}-\mathrm{LAC}_{\mathrm{AUC}}$ during the initial postoperative period than cows with $\mathrm{PO}$, indicating not only the extent but also the duration of hyper-L-lactatemia during the initial postoperative period as a negative prognostic factor. Taken together, these results confirm previous findings in calves (Lausch et al., 2020) and are in line with previous studies in horses with colitis and other abdominal emergencies (Tennent-Brown et al., 2010; Hashimoto-Hill et al., 2011). It also agrees with studies on dogs with gastric dilatation-volvulus (Zacher et al., 2010; Green et al., 2011) where a delayed return to normo-lactatemia or persistent hyperlactatemia during the initial therapeutic period was identified as a more reliable prognostic indicator than hyperlactatemia before initiation of therapy. However, in terms of the predictability of $\mathrm{NO}$, the results of the ROC analyses indicated that calculated values for L-LAC $\mathrm{AUC}_{\mathrm{AU}}$ and $\Delta_{\%} \mathrm{~L}-\mathrm{LAC}$ did not provide an advantage over absolute values for $\mathrm{L}$ LAC at the start or end of the respective time interval. This was also the case in our previous study in calves (Lausch et al., 2020). Therefore, we concluded that the prognostic information of postoperative L-LAC in cows is also not dependent on repeated consecutive measurement, which facilitates the applicability of our findings in the field.

Given that postoperative L-LAC measurements allow a more accurate prediction of the outcome of therapy, it can be speculated that hyper-L-lactatemia during the early postoperative period represents a more accurate indicator of ischemia-induced injuries and gastrointestinal organ damage, and that the relative effect of the underlying pathophysiological mechanisms for hyper-Llactatemia changed between the pre- and postoperative phases. Given that L-LAC has a relatively short plasma half-life of 20 to $60 \mathrm{~min}$ (Orringer et al., 1977; Pieschl et al., 1992; De Pedro et al., 2012), hyper-L-lactatemia, secondary to insufficient tissue oxygen supply (type A hyperlactatemia), should rapidly respond to volume repletion and surgical intervention. Therefore, postoperative hyperlactatemia was more likely related to dysmetabolism and poor oxygen utilization by tissues in the face of adequate oxygen delivery, which has been classified as type B hyperlactatemia in the literature (Pang and Boysen, 2007; Rosenstein et al., 2018). Possible mechanisms include reperfusion injury and inflammation in damaged tissues (Moore et al., 1981; Moore et al., 1994), as well as microcirculatory dysfunction and altered cellular respiration due to sepsis and endotoxemia (Fink, 2002; Ince, 2005).
Unfortunately, the predictive accuracy of postoperative plasma L-LAC in terms of the outcome after 3 mo was lower than in terms of the outcome until hospital discharge. This can be explained by the fact that a NO after discharge was exclusively related to a relapse in 4 cows that completely recovered until discharge, and consequently had a normalization of L-LAC values during their hospital stay. Three out of 4 of these cows originally suffered from hemorrhagic bowel syndrome, which agrees with a previous report where a relatively high recurrence rate for this condition (28\% during the first year after clinical discharge) was reported (Peek et al., 2009).

Although the present analyses provided valuable information in respect to the prognostic utility of pre- and postoperative L-LAC measurement in cows with acute abdominal emergencies, there were several shortcomings related to this study. These shortcomings include the preselection of cases that were admitted to a university teaching hospital, the potential effect of euthanasia on the outcome, and documentation issues related to retrospective analyses. Another potential limitation was the fluid-therapy protocol used for the prospective study population that was obviously not standardized to the BW level of individual cows. No significant association between the administered amounts of fluids and L-LAC and $\Delta_{\%} \mathrm{~L}-\mathrm{LAC}$ were seen at 6 and $12 \mathrm{~h}$. However, significant associations were seen at 24,48 , and $72 \mathrm{~h}$, which likely resulted from cows with signs of clinical dehydration receiving more intravenous fluids or a repeated oral treatment according to the protocol. The large variety of individual diagnoses of cows of the prospective study population might be considered as another limitation because it prevented any meaningful statistical analyses on the individual diagnosis level. A validation of the identified cut-off values in a distinct and potentially larger study population is therefore warranted and ideally should be performed in a field or on-farm setting. We suggest that future studies aim to assess whether multivariable models based on standardized clinical and other biochemical analyses provide a more accurate prediction of the outcome of therapy.

\section{CONCLUSIONS}

The present study confirms previous findings in calves with surgical abdominal emergencies that indicated that a delayed return to normo-L-lactatemia or persistent hyper-L-lactatemia during the initial postoperative period is a more reliable prognostic indicator than hyperlactatemia before initiation of surgery. In the present study, plasma L-LAC measurements at $6 \mathrm{~h}$ after start of surgical intervention provided the 
most accurate information in respect to the outcome until hospital discharge. Therefore, postoperative measurement of L-LAC should be included in the clinical management of cows with acute abdominal emergencies because it can help to identify cows with an increased risk for a NO at an early stage after surgical intervention that might benefit from close monitoring and more intensive treatment protocols.

\section{ACKNOWLEDGMENTS}

The authors thank all clinicians and staff members at the Clinic for Ruminants with Ambulatory and Herd Health Services (LMU Munich) who dealt with the clinical cases for their assistance. The authors have not stated any conflicts of interest.

\section{REFERENCES}

Adaska, J. M., S. S. Aly, R. B. Moeller, P. C. Blanchard, M. Anderson, H. Kinde, and F. Uzal. 2014. Jejunal hematoma in cattle: A retrospective case analysis. J. Vet. Diagn. Invest. 26:96-103. https: //doi.org/10.1177/1040638713517696.

Boulay, G., D. Francoz, E. Dore, S. Dufour, M. Veillette, M. Badillo, A. M. Belanger, and S. Buczinski. 2014. Preoperative cow-side lactatemia measurement predicts negative outcome in Holstein dairy cattle with right abomasal disorders. J. Dairy Sci. 97:212-221. https://doi.org/10.3168/jds.2013-6898.

Braun, U., T. Schmid, E. Muggli, K. Steininger, M. Previtali, C. Gerspach, A. Pospischil, and K. Nuss. 2010. Clinical findings and treatment in 63 cows with haemorrhagic bowel syndrome. Schweiz. Arch. Tierheilkd. 152:515-522. https://doi.org/10.1024/0036 $-7281 / a 000117$.

Buczinski, S., G. Boulay, and D. Francoz. 2015. Preoperative and postoperative L-lactatemia assessment for the prognosis of right abomasal disorders in dairy cattle. J. Vet. Intern. Med. 29:375-380. https://doi.org/10.1111/jvim.12490.

Buczinski, S., E. Dore, G. Boulay, and D. Francoz. 2014. Validation of the handheld Lactate-Pro analyzer for measurement of blood L-lactate concentration in cattle. Vet. Clin. Pathol. 43:567-572. https://doi.org/10.1111/vcp.12185.

Burfeind, O., and W. Heuwieser. 2012. Validation of handheld meters to measure blood L-lactate concentration in dairy cows and calves. J. Dairy Sci. 95:6449-6456. https://doi.org/10.3168/jds.2012-5329.

Constable, P. D., K. W. Hinchcliff, S. H. Done, and W. Grünberg. 2017. Veterinary Medicine: A Textbook of the Diseases of Cattle, Horses, Sheep, Pigs and Goats. 11th ed. Elsevier Health Sciences, St. Louis, MO.

Constable, P. D., G. St Jean, B. L. Hull, D. M. Rings, and G. F. Hoffsis. 1991. Preoperative prognostic indicators in cattle with abomasal volvulus. J. Am. Vet. Med. Assoc. 198:2077-2085.

Constable, P. D., G. St Jean, B. L. Hull, D. M. Rings, D. E. Morin, and D. R. Nelson. 1997. Intussusception in cattle: 336 cases (19641993). J. Am. Vet. Med. Assoc. 210:531-536.

De Pedro, P., P. A. Wilkins, M. A. McMichael, L. Dirikolu, K. M. Lascola, S. C. Clark-Price, and R. C. Boston. 2012. ExogenousLlactate clearance in adult horses. J. Vet. Emerg. Crit. Care (San Antonio) 22:564-572. https://doi.org/10.1111/j.1476-4431.2012 $.00800 . x$.

Elhanafy, M. M., D. D. French, and U. Braun. 2013. Understanding jejunal hemorrhage syndrome. J. Am. Vet. Med. Assoc. 243:352358. https://doi.org/10.2460/javma.243.3.352.

Fink, M. P. 2002. Bench-to-bedside review: Cytopathic hypoxia. Crit. Care 6:491-499. https://doi.org/10.1186/cc1824.
Fubini, S. L., Y. T. Grohn, and D. F. Smith. 1991. Right displacement of the abomasum and abomasal volvulus in dairy cows: 458 cases (1980-1987). J. Am. Vet. Med. Assoc. 198:460-464.

Green, T. I., C. C. Tonozzi, R. Kirby, and E. Rudloff. 2011. Evaluation of initial plasma lactate values as a predictor of gastric necrosis and initial and subsequent plasma lactate values as a predictor of survival in dogs with gastric dilatation-volvulus: 84 dogs (20032007). J. Vet. Emerg. Crit. Care (San Antonio) 21:36-44. https:// doi.org/10.1111/j.1476-4431.2010.00599.x.

Haji-Michael, P. G., L. Ladrière, A. Sener, J.-L. Vincent, and W. J. Malaisse. 1999. Leukocyte glycolysis and lactate output in animal sepsis and ex vivo human blood. Metabolism 48:779-785. https:// doi.org/10.1016/S0026-0495(99)90179-8.

Hashimoto-Hill, S., K. G. Magdesian, and P. H. Kass. 2011. Serial measurement of lactate concentration in horses with acute colitis. J. Vet. Intern. Med. 25:1414-1419. https://doi.org/10.1111/j.1939 $-1676.2011 .00808 . x$.

Ince, C. 2005. The microcirculation is the motor of sepsis. Crit. Care 9(Suppl. 4):S13-19. https://doi.org/10.1186/cc3753.

Karapinar, T., O. Kaynar, A. Hayirli, and M. Kom. 2013. Evaluation of 4 point-of-care units for the determination of blood L-lactate concentration in cattle. J. Vet. Intern. Med. 27:1596-1603. https:/ /doi.org/10.1111/jvim.12204.

Lausch, C. K., A. Lorch, S. Giertzuch, A. Rieger, G. Knubben-Schweizer, and F. M. Trefz. 2020. Prognostic relevance of pre- and postoperative plasma L-lactate measurements in calves with acute abdominal emergencies. J. Dairy Sci. 103:1856-1865. https://doi .org/10.3168/jds.2019-17224.

Lausch, C. K., A. Lorch, G. Knubben-Schweizer, A. Rieger, and F. M. Trefz. 2019. Prognostic value of preoperative plasma L-lactate concentrations in calves with acute abdominal emergencies. J. Dairy Sci. 102:10202-10212. https://doi.org/10.3168/jds.2019 $-16871$.

Meylan, M. 1999. Prognostic indicators in cattle with right-sided displacement of the abomasum and abomasal volvulus. Schweiz. Arch. Tierheilkd. 141:413-418.

Moore, J. N., N. A. White, J. N. Berg, C. M. Trim, and H. E. Garner. 1981. Endotoxemia following experimental intestinal strangulation obstruction in ponies. Can. J. Comp. Med. 45:330-332.

Moore, R. M., W. Muir, A. Bertone, and W. Beard. 1994. Characterization of the hemodynamic and metabolic alterations in the large colon of horses during low-flow ischemia and reperfusion. Am. J. Vet. Res. 55:1444-1453.

Orringer, C. E., J. C. Eustace, C. D. Wunsch, and L. B. Gardner. 1977. Natural history of lactic acidosis after grand-mal seizures. N. Engl. J. Med. 297:796-799. https://doi.org/10.1056/ NEJM197710132971502.

Pang, D. S., and S. Boysen. 2007. Lactate in veterinary critical care: Pathophysiology and management. J. Am. Anim. Hosp. Assoc. 43:270-279. https://doi.org/10.5326/0430270.

Peek, S. F., E. M. Santschi, M. A. Livesey, M. A. Prichard, S. M. McGuirk, S. H. Brounts, and R. B. Edwards III.. 2009. Surgical findings and outcome for dairy cattle with jejunal hemorrhage syndrome: 31 cases (2000-2007). J. Am. Vet. Med. Assoc. 234:13081312. https://doi.org/10.2460/javma.234.10.1308.

Pieschl, R. L., P. W. Toll, D. E. Leith, L. J. Peterson, and M. R. Fedde. 1992. Acid-base changes in the running greyhound: Contributing variables. J. Appl. Physiol. 73:2297-2304. https://doi .org/10.1152/jappl.1992.73.6.2297.

Rademacher, G., I. Lorenz, and T. Hänichen. 2002. Jejunumanschoppung mit koaguliertem Blut infolge blutender Darmulzera bei Kühen. Tierarztl. Umsch. 57:399-411.

Rosenberger, G. 1990. Die klinische Untersuchung des Rindes. Parey, Berlin, Germany.

Rosenstein, P. G., B. S. Tennent-Brown, and D. Hughes. 2018. Clinical use of plasma lactate concentration. Part 1: Physiology, pathophysiology, and measurement. J. Vet. Emerg. Crit. Care (San Antonio) 28:85-105. https://doi.org/10.1111/vec.12708.

Shimojo, N., K. Naka, C. Nakajima, C. Yoshikawa, K. Okuda, and K. Okada. 1989. Test-strip method for measuring lactate in 
whole blood. Clin. Chem. 35:1992-1994. https://doi.org/10.1093/ clinchem/35.9.1992.

Tennent-Brown, B. S., P. A. Wilkins, S. Lindborg, G. Russell, and R. C. Boston. 2010. Sequential plasma lactate concentrations as prognostic indicators in adult equine emergencies. J. Vet. Intern. Med. 24:198-205. https://doi.org/10.1111/j.1939-1676.2009.0419.x.

Trefz, F. M., I. Lorenz, A. Lorch, and P. D. Constable. 2017. Clinical signs, profound acidemia, hypoglycemia, and hypernatremia are predictive of mortality in 1,400 critically ill neonatal calves with diarrhea. PLoS One 12:e0182938. https://doi.org/10.1371/journal .pone.0182938.

Wilkins, P. A., B. J. Sheahan, K. A. Vander Werf, C. Castagnetti, J. Hardy, A. Schoster, and R. C. Boston. 2015. Preliminary investigation of the area under the L-lactate concentration-time curve (LACArea) in critically ill equine neonates. J. Vet. Intern. Med. 29:659-662. https://doi.org/10.1111/jvim.12559.
Youden, W. J. 1950. Index for rating diagnostic tests. Cancer 3:32-35. https://doi.org/10.1002/1097-0142(1950)3:1<32:AIDCNCR2820030106>3.0.CO;2-3.

Zacher, L. A., J. Berg, S. P. Shaw, and R. K. Kudej. 2010. Association between outcome and changes in plasma lactate concentration during presurgical treatment in dogs with gastric dilatation-volvulus: 64 cases (2002-2008). J. Am. Vet. Med. Assoc. 236:892-897. https://doi.org/10.2460/javma.236.8.892.

\section{ORCIDS}

S. Giertzuch ๑ https://orcid.org/0000-0002-0677-8855

C. K. Lausch (1) https://orcid.org/0000-0001-5350-4702

G. Knubben-Schweizer (® https://orcid.org/0000-0002-0928-5933

F. M. Trefz @ https://orcid.org/0000-0002-9784-8889 